\title{
A telessaúde como estratégia de resposta do Estado: revisão sistemática
}

\author{
Rafaela Santana Celes, ${ }^{1}$ Thaís Regis Aranha Rossi, ${ }^{2}$ Sandra Garrido de Barros, ${ }^{3}$ \\ Carla Maria Lima Santos ${ }^{2}$ e Carla Cardoso ${ }^{2}$
}

Como citar Celes RS, Rossi TRA, Barros SG, Santos CML, Cardoso C. A telessaúde como estratégia de resposta do Estado: revisão sistemática. Rev Panam Salud Publica. 2018;42:e84. https://doi.org/10.26633/ RPSP.2018.84

RESUMO Objetivo. Identificar ações de telessaúde descritas na literatura como estratégias de politicas nacionais de saúde.

Métodos. Foi realizado um estudo de revisão sistemática da produção científica sobre utilização da telessaúde como estratégia de resposta do Estado a problemas ou necessidades de saúde da população, utilizando-se as bases de dados Biblioteca Virtual em Saúde (BVS), PubMed e Google Acadêmico. Os termos utilizados na busca foram "telessaude politicas", "implantacao telessaude", "telehealth policy", "telehealth America", "telehealth Asia", "telehealth Antartida", "telehealth Europe", "telehealth Africa", "telehealth Oceania". A coleta de dados foi feita no período de março de 2016 a fevereiro de 2017.

Resultados. Foram analisados 21 artigos em português, espanhol e inglês sobre telessaúde em distintos países. Não houve concentração expressiva de artigos por local ou região. O maior número de publicações ocorreu de 2014 a 2017. A telessaúde tem sido implementada especialmente para diminuir custos na saúde, educação permanente de profissionais de saúde, interconsulta, fortalecimento da atenção primária à saúde e melhoria do acesso à saúde em áreas remotas.

Conclusões. A telessaúde é utilizada como política pública na Europa, Américas, Ásia e África, existindo, no entanto, variações em relação ao estágio de implantação. As principais diferenças quanto à telessaúde nos distintos países foram de infraestrutura, financiamento, engajamento de pacientes e cuidadores e posicionamento do Estado frente ao papel da telessaúde.

Palavras-chave Telemedicina; recursos em saúde; política de saúde.

A telessaúde consiste na utilização de tecnologias de informação e comunicação (TIC) para prestar serviços de saúde a

\footnotetext{
Universidade do Estado da Bahia, Programa de Residência Multiprofissional, Salvador (BA), Brasil.

2 Universidade do Estado da Bahia, Departamento de Ciências da Vida, Salvador (BA), Brasil. Correspondência: Thaís Regis Aranha Rossi, thais. aranha@gmail.com

3 Universidade Federal da Bahia (UFBa), Faculdade de Odontologia, Departamento de Odontologia Social e Pediátrica, Salvador (BA), Brasil
}

distância e para compartilhar informações e conhecimento. Na literatura, os termos telessaúde, telemedicina e eHealth são frequentemente utilizados; eles expressam estratégias de resposta para problemas de saúde socialmente construídos, como escassez de profissionais em áreas remotas e aumento da longevidade da população (1-3).

No século XIX, o desenvolvimento dos serviços postais, o uso do telégrafo e, posteriormente, o uso do telefone facilitaram a troca de informações e a divulgação das práticas médicas. No século XX, o uso da televisão e da radiocomunicação permitiram maior troca de informações de saúde. Com o advento da Internet, a partir da década de 1990, cresceu a integração da telecomunicação às necessidades de saúde (4). Atualmente, a telessaúde utiliza os mais diversos mecanismos, inclusive palestras por videoconferência 
para educação permanente e interconsultas, telefone, mensagens via celular, plataformas de mensagens via Internet, vídeos ou mensagens via satélite (1-10).

Diante desse histórico, tornou-se evidente a possibilidade de resultados nas ações de telessaúde para prevenção de doenças, promoção da saúde e tratamento de morbidades, justificando investimentos por parte dos governos. Levando em consideração esses aspectos, fizemos a seguinte pergunta de investigação: existem países com políticas públicas de telessaúde? Assim, este estudo teve como objetivo identificar na literatura artigos que descrevessem ações de telessaúde como parte de políticas públicas nacionais.

\section{MATERIAIS E MÉTODOS}

Foi realizado um estudo de revisão sistemática da produção científica sobre políticas de telessaúde como estratégia de resposta do Estado a problemas ou necessidades de saúde da população. Neste estudo, adotou-se como conceito de política de saúde aquele descrito por Paim, que se refere à "resposta social (ação ou omissão) de uma organização (como o Estado) diante das condições de saúde dos indivíduos e das populações e seus determinantes, bem como em relação à produção, distribuição, gestão e regulação de bens e serviços que afetam a saúde humana e o ambiente" (7, p. 588). O autor considera em seu conceito tanto as ações quanto as omissões do Estado perante problemas de saúde da população; neste artigo, entretanto, privilegiaram-se os trabalhos sobre ações, ou seja, as respostas do Estado, descritas em documentos publicados.

Foram pesquisadas as bases de dados Biblioteca Virtual em Saúde (BVS) (http://brasil.bvs.br), PubMed (https:/ / www.ncbi.nlm.nih.gov/m/pubmed) e Google Acadêmico (https://scholar. google.com.br). O período de coleta de dados foi de março 2016 a fevereiro de 2017, sendo selecionados 21 artigos publicados entre 2004 e 2017.

\section{Seleção dos artigos}

Foram utilizados como descritores: "telessaude politicas", "implantacao telessaude", "telehealth policy", "telehealth America", "telehealth Asia", "telehealth Antartida", "telehealth Europe", "telehealth Africa", "telehealth Oceania".
Não foram utilizadas acentuações ortográficas. Essa busca sem filtros identificou 12097 documentos, em língua inglesa, portuguesa, espanhola e em outros idiomas (alemão, italiano, russo, sueco, chinês). Em seguida, foi realizada uma filtragem para trabalhos em inglês, espanhol e português e, posteriormente, nova filtragem dos artigos com texto completo disponível. Nessa etapa, foram excluídos 9559 trabalhos (figura 1).

Os 2538 trabalhos restantes foram inicialmente analisados pelo título. Foram excluídos trabalhos em duplicata, editoriais, dissertações, teses, artigos que versavam sobre outros temas ou sobre telessaúde como estratégia restrita e privada, excluindo-se 2481 artigos. Foram lidos os resumos dos 57 artigos restantes. A partir dos mesmos critérios, foram selecionados 21 artigos para análise, sendo excluídos artigos que tratavam apenas de equipamentos tecnológicos utilizados na telessaúde. Não houve revisão manual das listas de referências.

Como procedimento de análise, os 21 artigos selecionados foram lidos na íntegra e classificados em uma planilha do Microsoft Excel 2010 com as variáveis ano, periódico, autor, objetivo, país, metodologia, local de publicação, continente, cidade/país, título, periódico, ano e principais achados dos artigos (tabela 1).

Na etapa seguinte, os resultados foram organizados em planilha Excel 2010 de acordo com a sequência dos achados na
FIGURA 1. Esquema de seleção de artigos sobre ações de telessaúde vinculadas a políticas públicas

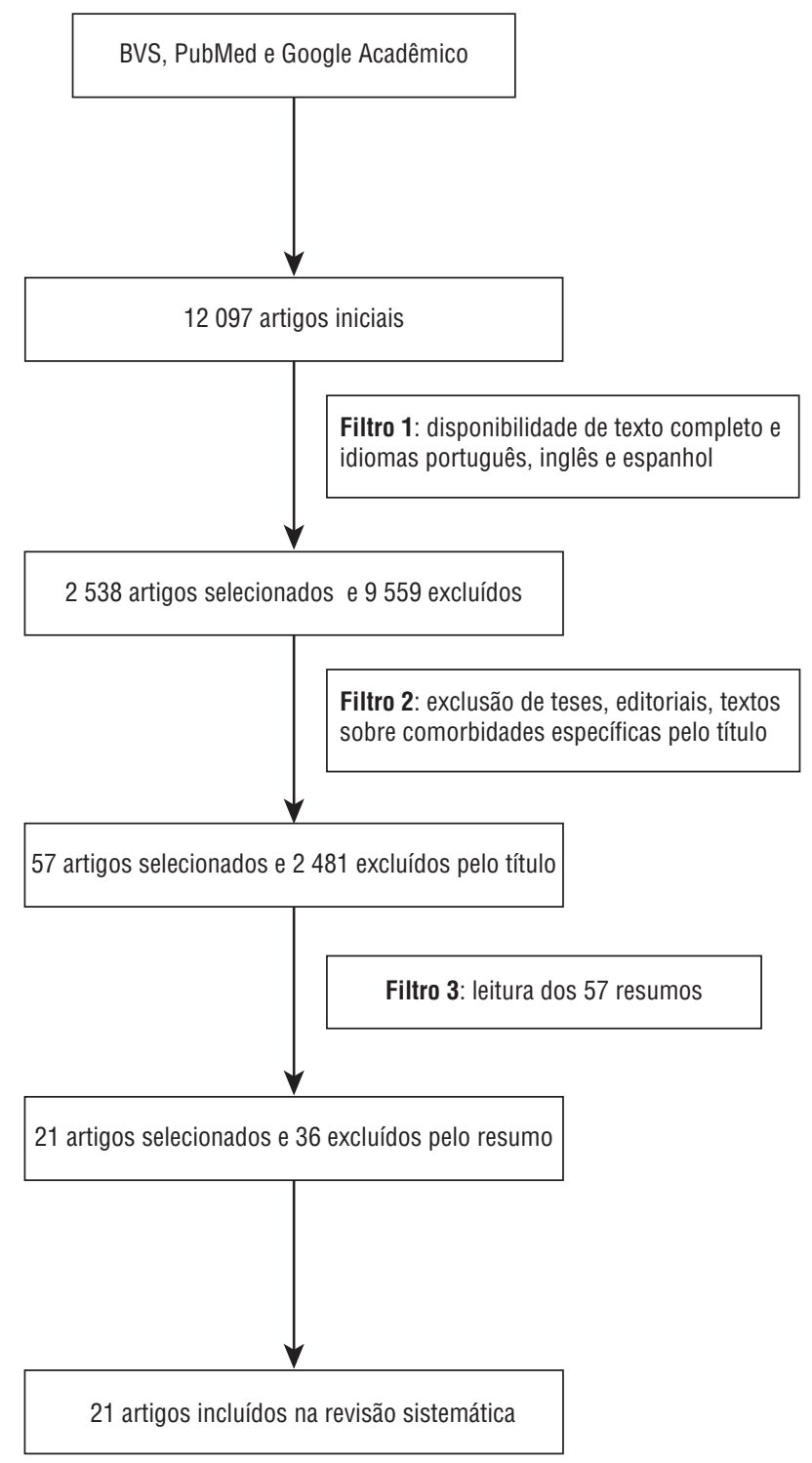


TABELA 1. Distribuição geográfica dos estudos e ações de telessaúde vinculadas a políticas públicas

\begin{tabular}{|c|}
\hline Origem (referência) \\
\hline África \\
\hline África (3) \\
\hline Quênia (11) \\
\hline África do Sul (12) \\
\hline África do Sul (13) \\
\hline Américas \\
\hline Brasil (2) \\
\hline Brasil (1) \\
\hline Colômbia (15) \\
\hline $\begin{array}{l}\text { Bolívia, Costa Rica, Cuba, } \\
\text { El Salvador, Guatemala, Peru, } \\
\text { Venezuela, Brasil, Colômbia, } \\
\text { Equador, México e Panamá (14) }\end{array}$ \\
\hline Estados Unidos (6) \\
\hline Ásia \\
\hline Coreia (6) \\
\hline Malásia (10) \\
\hline Bangladesh (18) \\
\hline $\begin{array}{l}\text { Bangladesh, Índia, Indonésia, } \\
\text { Maldivas, Malásia, Filipinas, } \\
\text { Sri Lanka (16) }\end{array}$ \\
\hline $\begin{array}{l}\text { Japão, Taiwan, Coreia } \\
\text { do Sul (17) }\end{array}$ \\
\hline Japão $(6,19)$ \\
\hline
\end{tabular}

Europa (6)

Itália (23)

Espanha, Itália, Áustria,

França, Dinamarca (21)

Alemanha (6)

Escócia (22)

Reino Unido (9)

Reino Unido (20)

Dinamarca, Estônia, Franca, Alemanha, Itália, Países baixos, Espanha, Reino Unido (8) Oceania

Austrália (24)

Ano $\quad$ Principais achados

2017 A TIC é incipiente na África. Fatores como cultura religiosa, falta de conhecimento, influenciam o sistema de saúde. A telessaúde na África é escassa e pobre em infraestrutura.

2017 As estratégias de telessaúde no Quênia estão concentradas em atenção primária e Aids. Número pequeno de projetos localizados em áreas marginalizadas e periféricas.

2011 Em 1998, o governo sul-africano iniciou a primeira fase do Projeto Nacional de Telemedicina, mas fracassou. 0 artigo analisa as ações desta iniciativa nacional e os motivos de fracasso. Também apresenta a o caso do projeto de tele-educação de uma Universidade que vem desenvolvendo distintas ações com parceria governamental para a África do Sul.

2014 Enquanto 0 uso da telemedicina na África é restrito, existe um número considerável de programas de tele-educação. Apesar de ter sido lançado, em 1999, um projeto nacional em telessaúde na África do Sul, nem a segunda nem a terceira fases foram concluídas.

2014 A utilização da teleodontologia tem sido eficiente e serve como ferramenta de apoio técnico assistencial, reduzindo número de encaminhamentos, ampliando o acesso dos profissionais a educação permanente, evitando deslocamentos no Sistema Único de Saúde.

2008 A telemedicina vem se consolidando no Brasil com ações governamentais e com apoio das agências de fomento à pesquisa e iniciativas que possibilitaram a formação de equipes e núcleos de pesquisa em diversas instituições universitárias brasileiras. Retrata projetos de comunicação em saúde, educação à distância, educação permanente para profissionais da área de saúde.

2013 A Colômbia conta, desde 2012, com o Sistema Nacional de Informação em Câncer, com ações políticas e estratégias para implementar essas novas tecnologias na saúde.

2014 Bolívia, Costa Rica, Cuba, El Salvador, Guatemala, Peru e Venezuela estão em processo de elaboração e início de implantação de programas. Brasil, Colômbia, Equador, México e Panamá mantêm projetos nacionais de telessaúde.

2015 Projetos para fornecer serviços médicos a cidadãos de áreas remotas. Serviços médicos conectados para oferecer telemedicina aos pacientes. Além das interconsultas, são realizados tratamentos com a tecnologia da Internet e HIT. Os projetos continuam em expansão.

2015 Na Coreia, é utilizada no tratamento de doenças crônicas, idosos e portadores de deficiência, mas não há uma legislação específica para telemedicina.

20150 Ministério da Saúde da Malásia incorporou a telessaúde como política nacional desde 1990, seguindo a tendência de vários países, em resposta aos altos custos em saúde.

2014 Bangladesh carece de preparação e planejamento para sustentar a telessaúde.

2015 Foram identificados sete programas nacionais de telessaúde na Índia, Bangladesh, Malásia, Maldivas, Filipinas, Sri Lanka e Indonésia. Essas políticas estão em diferentes estágios de implementação. A telessaúde na região trouxe melhorias da atenção à saúde, equidade, melhor acesso às informações.

20090 uso da telemedicina aumentou a qualidade do cuidado à saúde e 0 acesso aos serviços. Porém, o custo compromete a sustentabilidade da telemedicina. 0 sucesso dos programas não depende só de boa tecnologia, mas de organização dos recursos humanos.

2011 A telemedicina tem auxiliado as expedições japonesas à Antártida, permitindo comunicação entre pacientes e médicos na Antártida e no Japão. Os casos mais comuns são dermatites, fraturas, doenças respiratórias devido ao clima frio e ao gelo (19). No Japão, a telemedicina é utilizada por pacientes em casa que usam oxigênio, apresentam doenças crônicas e incuráveis. É utilizada como complemento ao tratamento convencional (6).

2015 Na União Europeia é relatada uma variedade de programas para gerenciamento de doenças, assim como sua utilização para teleconsultas e em serviços assistenciais.

2004 A telessaúde tem sido utilizada para promover saúde em expedições italianas na Antártida.

2015 A telessaúde pode contribuir para superar as restrições espaciais e temporais. Afirma que o empoderamento dos pacientes para auto-gestão e tomada de decisão compartilhada tem sido importante na utilização da telessaúde em nove regiões europeias.

2015 Implementação de programa para monitoramento de pacientes.

20110 estudo indicou que as iniciativas de telessaúde são bem-vindas por pacientes e cuidadores. Afirma que a telessaúde deve ser uma modalidade complementar ao sistema clínico tradicional, não uma alternativa.

2016 As principais barreiras que influenciaram a utilização da telessaúde foram falta de informações sobre o serviço e equipamentos disponíveis, falta de experiência e confiança para usar o sistema, estigma do uso. Facilitadores foram a simplicidade de uso e a confiabilidade da telessaúde.

2017 Fatores interferem no sucesso da telessaúde: macro (mercado, infraestrutura, política), meso (organizacional) e micro (profissional ou público). Os fatores que impediram a implementação foram: falta de tecnologia, incerteza sobre governança da informação, falta de incentivos.

20130 estudo identificou oito facilitadores para a telessaúde: reorganização de serviços, foco no paciente, mecanismos de governança, sistemas de informação interoperáveis, compromisso político, profissionais engajados, investimentos nacionais e programas de financiamento e incentivos e financiamento.

Os serviços de telessaúde mental na Austrália são mais utilizados por mulheres e aqueles de condição socioeconômica e educação 2017 elevadas. Entre os usuários, existia uma preferência por tratamentos presenciais em relação à telessaúde. Cita-se a utilização em teleconsultas, utilização e desenvolvimento de programas, terapias on-line, atendimento de saúde mental moderado por médicos. 
matriz. Esse processo foi desenvolvido por uma das pesquisadoras (RSC) e confirmado por outra (TRAR). Os artigos selecionados foram lidos na íntegra pelas duas pesquisadoras. Inicialmente, procedeu-se a uma análise descritiva, com vistas à compreensão das publicações sobre o tema. Em seguida, buscou-se identificar como essas políticas vêm sendo formuladas e implementadas, organizadas por continentes, para que fosse possível apresentar um panorama mundial.

\section{RESULTADOS}

Foram analisados 21 artigos sobre a telessaúde em 40 países (tabela 1): três países do continente Africano (3, 11-13), 13 das Américas (1, 2, 14, 15), 12 da Ásia (6, 10, 16-19), 11 da Europa (8, 9, 20-22) e um da Oceania (23). Não houve concentração expressiva de artigos por local ou região (tabela 1). Na África e nas Américas, os estudos indicam incipiência na utilização da telessaúde, muitos países estando em fase de implantação de serviços (Bolívia, Costa Rica, Cuba, El Salvador, Guatemala, Peru, Venezuela, África e África do Sul). Por outro lado, países como Brasil, Colômbia, Equador, México e Panamá já mantêm projetos de telessaúde. Na Ásia, também há diferentes estágios de implementação de projetos de telessaúde, mas já há evidências de melhoria da atenção à saúde, equidade e acesso à informação. Na Europa, pode-se verificar um estágio mais avançado de implantação dos projetos de telessaúde, inclusive com estudos sobre barreiras de utilização e fatores facilitadores de sucesso e fracasso. Na Austrália, um artigo avaliou a utilização de serviços de telessaúde em comparação aos serviços presenciais de saúde. Também foram encontrados artigos descrevendo a utilização da telessaúde no apoio a expedições japonesas e italianas à Antártica (tabela 1).

Nos artigos levantados neste estudo, a telessaúde foi utilizada especialmente para a educação permanente de profissionais de saúde (13), para redução de custos em saúde $(6,10)$ e ampliação de acesso em áreas remotas $(6,14)$, cuidado hospitalar (13), atendimento a pacientes acamados e com restrição de locomoção (6), cuidados paliativos (22), serviços de educação permanente para estudantes, médicos, enfermeiras e parteiras, como pediatria, telerradiologia, telessonografia pré-natal, telepatologia, teleoftalmologia, dentre outros (13), teleodontologia
(14), melhorias e fortalecimento da atenção primária à saúde (APS) (14), integração de redes de atenção à saúde (14), melhoria da interconsulta (14), colaborações entre universidades e serviços de saúde (14) e melhoria dos registros nos sistemas de informação em saúde para subsidiar o planejamento de ações e serviços de saúde $(14,15)$.

Quanto aos tipos de estudo, grande parte tratava-se de relatos de experiência $(1,2,8,12,15,19,21,24)$ revisões de literatura $(4,13,16)$, revisões documentais $(6,10)$ e sistemáticas $(3,5,11,17,23)$, estudos de avaliação $(9,14,20,22)$, análise de política (10) e estudo de caso (18) (tabela 2). Um maior número de publicações foi observado de 2014 a 2017.

\section{DISCUSSÃO}

Os artigos identificados nesta revisão mostraram que pesquisadores de distintas localidades têm compartilhado com a comunidade científica experiências em telessaúde, revelando entraves e fatores positivos das ações implementadas. Essa colaboração auxilia gestores, formuladores de políticas e profissionais a compreenderem como têm se dado a implementação e a utilização da telessaúde em diferentes localidades e contextos. Entretanto, o fato de alguns autores não detalharem mais especificamente as diferentes estratégias usadas para implementação das ações consiste numa lacuna.

A ocorrência de um número maior de publicações nos anos de 2014 a 2017 evidenciou interesse crescente dos pesquisadores pelo assunto. Tal fato pode estar associado a fatores como larga utilização da Internet no século XXI (4), maior utilização e aproximação entre setores da comunicação, informação e saúde em vários países $(2,10)$, necessidade de diminuir

TABELA 2. Distribuição dos estudos segundo classificação de tipo de estudo e período de publicação

\begin{tabular}{|c|c|c|c|c|c|}
\hline \multirow{2}{*}{ Tipo de estudo (referência) } & \multicolumn{3}{|c|}{ Período } & \multirow{2}{*}{ Total } & \multirow{2}{*}{$\%$} \\
\hline & 2004-2008 & $2009-2013$ & 2014-2017 & & \\
\hline Análise de politica (18) & - & - & 1 & 1 & 4,76 \\
\hline Avaliação $(9,14,21,23)$ & - & 1 & 3 & 4 & 19,05 \\
\hline Estudo de caso (18) & - & - & 1 & 1 & 4,76 \\
\hline Relato de experiência $(1,2,8,12,15,19,20,22)$ & 2 & 3 & 3 & 8 & 38,10 \\
\hline Revisão documental (6) & - & - & 1 & 1 & 4,76 \\
\hline Revisão de literatura $(13,16)$ & - & & 2 & 2 & 9,52 \\
\hline Revisão sistemática $(3,11,17,24)$ & - & 1 & 3 & 4 & 19,05 \\
\hline Total $(\%)$ & $2(9,53)$ & $5(23,81)$ & $14(66,66)$ & 21 & 100,00 \\
\hline
\end{tabular}

gastos com tratamentos em saúde (9), aumento da expectativa de vida da população e necessidade de expansão dos serviços para áreas periféricas $(4,6,11)$.

Os artigos apontaram que a telessaúde pode ser uma estratégia para melhorar a atenção em saúde na África (11-13). O propósito inicial da telessaúde nesse continente era atuar na APS e no combate a Aids, ainda um grande problema de saúde pública na região (17). Dos projetos implementados, poucos estavam localizados em áreas marginalizadas, menos urbanizadas e com necessidades maiores de cuidado em saúde (10), onde os médicos eram escassos, a infraestrutura de saúde inadequada e as estratégias de tecnologia da informação e comunicação incipientes (3); de modo que deveria haver maior ênfase na implantação dessas estratégias nessas localidades.

$\mathrm{Na}$ África do Sul, as estratégias de telessaúde estavam concentradas no cuidado hospitalar (13). A primeira fase do projeto foi lançada em 1998 em 28 hospitais públicos, com serviços de telerradiologia, telessonografia pré-natal, telepatologia e teleoftalmologia, entre outros. O projeto fracassou por questões relacionadas a orçamento limitado, falta de gerenciamento e Internet limitada $(12,13)$. Apesar disso, alguns médicos, em parceria com universidades, lançaram projetos em províncias, com utilização de teleortopedia, telepsiquiatria e telerradiologia $(12,13)$.

O fator cultural e educacional da população é importante na utilização da telessaúde. Enquanto em países da África o misticismo e a religião interferem na aceitação de novas tecnologias e no cuidado em saúde (3), em países como Austrália (23), Escócia (22), Reino Unido (9) e outras regiões europeias (21), por questões culturais, os usuários e profissionais de saúde aceitam com maior facilidade a 
utilização da telessaúde. Nesse contexto, é importante o diálogo com os pacientes acerca dessa tecnologia a fim de mostrar os benefícios que ela pode trazer ao tratamento $(3,6,12,13,22,24)$.

Enquanto o uso da telessaúde como alternativa assistencial é restrito na África, a utilização da tele-educação é significativa, com iniciativas para educação permanente de trabalhadores de saúde e formação de estudantes (13). Além disso, seria interessante promover a integração dos setores saúde e educação, com vistas ao desenvolvimento de ações de educação em saúde voltada para a população.

Na América Latina, há diversas iniciativas (14) com objetivos distintos. No México, o principal intuito do projeto nacional de telessaúde, criado em 1995, foi contribuir para a universalidade no cuidado à saúde. Na Costa Rica, Peru e Venezuela, os projetos visavam a reduzir distâncias, favorecendo o acesso à saúde em áreas mais remotas (14), assim como ocorreu também na Coreia (6). No Equador e no Brasil, a ênfase estava na melhoria e fortalecimento da APS, assim como no continente africano (17). Outra iniciativa nacional no Brasil consiste numa teleconsultoria gratuita para médicos de todo país para discussão de casos clínicos, com ações de telediagnóstico e tele-educação (25). Destacam-se também as ações desenvolvidas no estado do Rio Grande do Sul, que vem servindo de referência para outras ações nacionais, como a realização de consultas clínicas com vistas a melhorar a qualidade do atendimento e agilizar o fluxo de usuários entre os níveis de atenção $(25,26)$. Outro projeto pioneiro em telessaúde ocorreu em Belo Horizonte, a partir de 2004, com a realização de teleconsultas (27). A teleodontologia também vem avançando como ferramenta de apoio técnico assistencial, contribuindo para redução do número de encaminhamentos, ampliando o acesso dos profissionais à educação permanente, evitando deslocamentos de pacientes no sistema público de saúde $(2,14)$.

Na Colômbia, o objetivo era melhorar as condições de saúde nos casos de vulnerabilidade; em El Salvador, integrar as redes de saúde; e na Guatemala, melhorar a interconsulta e desenvolver uma aplicação da telessaúde para o processo de interconsulta articulado com o registro básico de informações do paciente (14).

Ainda na Colômbia, vale citar a experiência com o Sistema Nacional de Informação do Câncer (SICAN), tendo em vista que seus autores compreendem a telessaúde como utilização das TIC nos serviços de saúde e com função relacionada a vigilância em saúde (15). O programa foi implementado em 2012 com a finalidade de organizar os dados dos pacientes para identificar informações necessárias para o planejamento dos serviços de saúde, para a investigação das causas e compreensão dos determinantes do câncer (15).

No continente asiático, foram identificados sete países com programas nacionais de telessaúde: Bangladesh, Índia, Indonésia, Malásia, Maldivas, Filipinas e Sri Lanka, com variações no estágio de desenvolvimento das políticas em cada país. A telessaúde vem sendo praticada, desde a década de 1990, pela Coreia, Japão, Estados Unidos e União Europeia (6). Na Coreia, a Lei Médica, de 2002, ampliou a área de atuação da telemedicina para tratamento de pacientes crônicos, idosos e portadores de deficiência e cidadãos que vivem em ilhas e áreas remotas.

No Japão, as bases iniciais foram lançadas, em 1997, pelo Departamento de Saúde e Serviços Humanos. As estratégias de telemedicina eram divididas em duas categorias: médico-médico e médico-paciente. Concentravam-se em pacientes que estavam em casa, faziam uso de oxigênio, doenças crônicas ou incuráveis, hipertensão, diabetes, dermatite atópica ou escaras.

Foi verificada a utilização da telemedicina também em expedições japonesas e italianas na Antártida $(6,22)$. No contexto da ausência de profissionais de saúde na Antártida, os médicos lá localizados mantinham contato com médicos no Japão, que orientavam tratamento de fraturas, dermatites, doenças respiratórias por vídeos e mensagens via satélite $(6,7)$. Assim, a telessaúde contribuiu para superar as restrições espaciais e temporais. $\mathrm{O}$ empoderamento dos pacientes para autogestão e tomada de decisão compartilhada também foi importante na utilização da telessaúde em nove regiões europeias (21).

Um estudo apontou que, na Malásia, o Ministério da Saúde incorporou a telessaúde como política nacional desde 1990, com o objetivo de reduzir os altos custos envolvendo saúde e melhorar a custoefetividade da utilização da comunicação e informação na saúde. Dessa forma, o uso da telessaúde teve como resultado o aumento da qualidade da educação em saúde e do tratamento a distância naquele país (10). Da mesma forma, na União Europeia, com o aumento das doenças crônicas, despesas médicas e envelhecimento da população, a telessaúde tem sido utilizada com a intenção de diminuir os custos e lidar com número limitado de médicos (6). O estudo de Lennon et al. (20) apontou uma boa regulamentação acerca da confidencialidade de informações de pacientes no Reino Unido, onde as estratégias de telessaúde têm sido utilizadas no cuidado de idosos e tratamento de doenças crônicas $(8,9)$.

Alguns autores $(8,9,20)$ dialogam no sentido de elencar fatores que promovem ou impedem a utilização da telessaúde na Europa, que poderiam ser estendidos para outros países. O estudo de Lennon et al. (20) identificou três níveis principais de questões que influenciam a saúde digital: macro (mercado, infraestrutura, política), meso (organizacional) e micro (profissional ou público). Os principais fatores que impediram a implementação foram: falta de tecnologia, incerteza acerca da governança da informação e falta de incentivos para priorizar a interoperabilidade (20). Fatores facilitadores para a implementação da telessaúde foram citados por Lennon et al. (20) e Villalba et al. (8): o corpo clínico, os defensores da saúde digital e a disposição pública e profissional (20), reorganização dos serviços, foco no paciente, mecanismos de governança, sistemas de informação interoperáveis, compromisso político, profissionais engajados e investimentos nacionais (8).

Um estudo com usuários e não usuários de telessaúde em Cambridgeshire, Reino Unido, especificou as principais barreiras para a utilização da telessaúde: falta de informações sobre o serviço e equipamentos disponíveis, falta de experiência e confiança para usar o sistema, estigma do uso (9). Como facilitadores, foram relacionadas a utilização e a confiabilidade da telessaúde $(8,9,20)$.

Na Escócia, o uso da telessaúde teve como objetivo os cuidados paliativos. As iniciativas foram bem aceitas por pacientes e cuidadores. Destaca-se que essas práticas são complementares à prática clínica, não alternativas a esta (22).

Na Austrália, esses serviços são mais utilizados por mulheres e por aqueles de boa condição socioeconômica e maior nível educacional. Entre os usuários, existia uma preferência por tratamentos presenciais em relação à telessaúde. Segundo o estudo, poucas pesquisas se 
concentraram no desenvolvimento de políticas e planejamento de implementação (23).

O presente estudo apresenta limitações que devem ser mencionadas. Apenas estudos publicados em português, inglês ou espanhol foram incluídos, o que possivelmente resultou na exclusão de relatos sobre o assunto. Além disso, não foram pesquisados documentos oficiais de políticas públicas de telessaúde, sendo que a publicação de artigos sobre tais políticas depende de interesses acadêmicos que por vezes não acompanham as políticas governamentais. Assim, os resultados não apresentam um retrato fidedigno da políticas nacionais de telessaúde. Entretanto, foi possível identificar iniciativas em diferentes continentes e mostrar um panorama global, ainda que superficial, das políticas de telessaúde.

Em conclusão, os artigos analisados permitiram identificar a existência de políticas nacionais de telessaúde na Europa, Américas, Ásia e África, em países como México, Costa Rica, Equador, Peru, Colômbia, El Salvador, Guatemala, Venezuela, Brasil, Bangladesh, Índia, Indonésia, Malásia, Maldivas, Filipinas, Sri Lanka, Reino Unido, Dinamarca, Estônia, França, Alemanha, Estados Unidos, Japão, África do Sul e Quênia. Os estágios de desenvolvimento dessas políticas variam de acordo com fatores políticos, econômicos, estruturais e culturais. Os estudos apontaram que essas políticas surgiram em resposta aos problemas de saúde da população, mas também como uma alternativa ao alto custo dos tratamentos, aumento das doenças crônicas, longevidade da população, falta de profissionais nas áreas remotas e periféricas. Foi possível observar que a telessaúde vem crescendo como resposta do Estado à construção de resposta aos problemas sociais.

Em relação aos projetos nacionais, o planejamento e a operacionalização desses projetos são importantes para que haja legislação específica e recursos financeiros e humanos destinados à implementação da telessaúde nos países. A integração dos serviços de saúde com o aparato tecnológico mostrou-se um agente facilitador no processo de utilização da telessaúde (8).

É consenso nos estudos que as áreas remotas, rurais e distantes dos centros urbanos são as que mais necessitam de investimentos. Por esse motivo, é necessária uma maior atenção da gestão em saúde para essas áreas a fim de implementar projetos de telessaúde, contribuindo para um maior acesso a serviços de saúde nessas áreas.

Tendo em vista os estudos analisados, compreende-se que a telessaúde, enquanto estratégia, tem um significativo potencial para a ampliar e qualificar o acesso à atenção à saúde, à educação permanente e na promoção da qualidade de vida da população nas diferentes localidades. A implementação de políticas nacionais e legislações específicas foram importantes para o progresso das iniciativas de telessaúde nos diferentes países.

Agradecimentos. RSC foi aluna do Programa de Residência Multiprofissional em Saúde na Universidade do Estado da Bahia, com bolsa de estudo concedida pelo Ministério da Saúde durante o período da pesquisa.

Conflitos de interesse. Nada declarado pelas autoras.

Declaração. As opiniões expressas no manuscrito são de responsabilidade exclusiva dos autores e não refletem necessariamente a opinião ou políticas da RPSP/PAJPH ou da Organização Pan-Americana da Saúde (OPAS).

\section{REFERÊNCIAS}

1. Wen CL. Telemedicina e telessaúde: um panorama no Brasil. Informat Pub. 2008; 10(2):7-15.

2. Correia AD, Dobashi BF, Gonçalves CC, Monreal VR, Nunes EA, Haddad PO, et al. Teleodontologia no programa nacional telessaúde Brasil redes: relato da experiência em Mato Grosso Do Sul. Rev ABENO. 2014;14(1):17-29.

3. Okoroafor II, Chukwuneke FN, Ifebunandu N, Onyeka TC, Ekwueme CO, Agwuna KK. Telemedicine and biomedical care in Africa: prospects and challenges. Niger J Clin Pract. 2017;20(1):1-5.

4. Craig J, Patterson V. Introduction to the practice of telemedicine. J Telemed Telecare. 2005;11:3-9.

5. Ekeland A, Bowes A, Flottorp S. Effectiveness of telemedicine: A systematic review of reviews. Int J Med Inform. 2010;79(11): 736-71.

6. Oh JY, Park YT, Jo EC, Kim SM. Current status and progress of telemedicine in Korea and other countries. Health Inform Res. 2015;21(4):239-43.

7. Paim J. Políticas de saúde no Brasil. Em: Rouquairol MZ, Almeida-Filho N, eds. Epidemiologia e saúde. Rio de Janeiro: Medsi; 2003. Pp. 587-603.

8. Villalba E, Casas I, Abadie F, Lluch M. Integrated personal health and care services deployment: experiences in eight European countries. Int J Med Inform. 2013;82(7): 626-35.

9. Cook E, Randhaw, G, Sharp C, Ali N, Guppy A, Barton G, et al. Exploring the factors that influence the decision to adopt and engage with an integrated assistive telehealth and telecare service in Cambridgeshire, UK: a nested qualitative study of patient 'users' and 'non-users'. BMC Health Serv Res. 2016;16:137.

10. Marzuki N, Ismail S, Al-Sadat N, Ehsan FZ, Chan $\mathrm{CK}, \mathrm{Ng} \mathrm{CW}$. Integrating information and communication technology for health information system strengthening: a policy analysis. Asia Pac J Public Health. 2015;27 (8 Suppl):86S-93S.

11. Njoroge M, Zurovac D, Ogara EA, Chuma J, Kirigiaet D. Assessing the feasibility of eHealth and mHealth: a systematic review and analysis of initiatives implemented in Kenya. BMC Res Notes. 2017;10:90.

12. Mars M. Telerehabilitation In South Africa - is there a way forward?. Int J Telerehabil. 2011;3(1):11-8.

13. Mars M. Tele-education in South Africa. Front Public Health. 2014;2:173.

14. Santos A, D’Agostino M, Brouskela M, Fernandéz A, Messina LA, Alves HJ. Uma visão panorâmica das ações de telessaúde na América Latina. Rev Panam Salud Publica. 2014;35(5/6):465-70.

15. Rivillas JC, Quintero JA, Caicedo J, Martínez M. Progresos en salud en Colombia: adopción del Sistema de Información Nacional en Cáncer. Rev Panam Salud Publica. 2014;35(5/6):446-52.

16. Marcelo A, Mohan J, Ganesh J, Kadam DB, Ratta BS, Kulatunga G, et al. Governance and management of national telehealth programs in Asia. Global Telehealth. 2015; 209:95-101.

17. Duranni H, Khoja S. A systematic review of the use of telehealth in Asian countries. J Telemed Telecare. 2009;15:175-81.

18. Ahmed T, Lucas $H$, Khan AS, Islam R, Bhuiya A, Iqbal M. eHealth and mHealth initiatives in Bangladesh: A scoping study. BMC Health Serv Res. 2014;14:260.

19. Ohno G. Practical results of telemedicine system between Antarctic Station and Japan. Em: Graschew G, ed. Telemedicine techniques and applications. Rijeka/ Shanghai: InTech; 2011. Pp. 439-53.

20. Lennon MR, Bouamrane MM, Devlin AM, O'Connor S, O'Donnell C, Chetty U, et al. Readiness for delivering digital health at scale: lessons from a longitudinal qualitative evaluation of a national digital health innovation program in the United Kingdom. J Med Internet Res. 2017;19(2):e42. 
21. Lettieri E, Fumagalli L, Radaelli G, Bertele P, Vogt J, Hammerschmidt R, et al. Empowering patients through ehealth: a case report of a pan-European project. BMC Health Serv Res. 2015;15:309.

22. Johnston B, Kidd L, Wengstrom $Y$, Kearney N. An evaluation of the use of Telehealth within palliative care settings across Scotland. J Palliat Med. 2012;26(2): 152-61.

23. Meurk C, Leung J, Hall W, Head BW, Whiteford $\mathrm{H}$. Establishing and governing e-mental health care in Australia: a systematic review of challenges and a call for policy-focussed research. J Med Internet Res. 2016;18(1):e10.

24. Pillon S, Todini AR. eHealth in Antarctica: a model ready to be transferred to every-day life. Int J Circumpolar Health. 2004;63(4): 436-42.

25. Gonçalves MR, Umpierre RN, D'Avila OP, Katz N, Mengue SS, Siqueira ACS, et al. Expanding primary care access: a telehealth success story. Ann Fam Med 2017; 15:383.

26. Harzheim E, Gonçalves MR, Umpierre RN, Siqueira ACS, Katz N, Agostinho MR, et al. Telehealth in Rio Grande do Sul, Brazil: bridging the gaps. Telemed J E Health. 2016;22(11):938-44. Epub 2016 Apr 20.

27. Melo MCB, Nunes MV, Resende RF, Figueiredo RR, Ruas SSM, Santos AF, et al. Telemed J E Health. 2017. doi: 10.1089/ tmj.2017.0165. [Epub ahead of print].

Manuscrito recebido em 10 de novembro de 2017 Aceito em versão revisada em 7 de abril de 2018 .
ABSTRACT

\section{Telehealth as state response strategy: systematic review}

Objective. To identify telehealth initiatives described in the literature as a strategy for national health policies.

Method. A systematic review was performed to identify articles focusing on the use of telehealth as a state response strategy to health problems or needs. The Virtual Health Library, PubMed, and Google Scholar were searched using the following keywords: "telessaude politicas", "implantacao telessaude", "telehealth policy", "telehealth America", "telehealth Asia", "telehealth Antartida", "telehealth Europe", "telehealth Africa", "telehealth Oceania". Data collection was performed from March 2016 to February 2017.

Results. Twenty-one articles describing telehealth initiatives in various countries, published in Portuguese, Spanish, and English, were analyzed. Concentration of studies on specific areas or regions was not detected. Most articles were published from 2014 to 2017. Telehealth initiatives have been used mainly to decrease health costs, for continued education of health care professionals, consultations between health care professionals, to strengthen primary health care, and to improve the access to health care in remote areas.

Conclusions. Telehealth is used as state policy across the five continents, with variations in the degree of implementation. The main differences in telehealth among countries refer to infrastructure, financing, engagement of patients and caretakers, and position of the state regarding the role of telehealth.

Keywords Telemedicine; health resources; health policy. 
RESUMEN Objetivo. Determinar las acciones de telesalud descritas en las publicaciones pertinentes como estrategia en materia de políticas nacionales de salud.

Método. Se realizó un estudio de revisión sistemática de la producción científica sobre la utilización de la telesalud como estrategia de respuesta del Estado a los

La telesalud como estrategia de respuesta del Estado: revisión sistemática problemas o a las necesidades de salud de la población, en el cual se emplearon las bases de datos de la Biblioteca Virtual de Salud (BVS), PubMed y Google Académico. Los términos utilizados en la búsqueda fueron "telesalud política", "implantación telesalud", "telehealth policy", "telehealth America", "telehealth Asia", "telehealth Antarctica", "telehealth Europe", "telehealth Africa" y "telehealth Oceania". Los datos se recopilaron entre marzo del 2016 y febrero del 2017.

Resultados. Se analizaron 21 artículos en español, inglés y portugués sobre telesalud en distintos países. No hubo ninguna concentración importante de artículos por lugar ni región. El mayor número de publicaciones se registró entre el 2014 y el 2017. La estrategia de telesalud se ha puesto en práctica para reducir los costos de la atención de salud, fomentar la educación permanente de los profesionales de salud y facilitar las consultas entre ellos, fortalecer la atención primaria de salud y ampliar el acceso a los servicios de salud en las zonas remotas.

Conclusiones. La estrategia de telesalud se utiliza como política pública en África, América, Asia y Europa, pero existen variaciones con respecto a la fase de implantación. Las principales diferencias en materia de telesalud observadas en los distintos países correspondieron a infraestructura, financiamiento, compromiso de los enfermos y prestadores de cuidado, y postura del Estado frente al papel de la telesalud.

Palabras clave Telemedicina; recursos en salud; política de salud. 